\title{
Interactive comment on "Exploring the Long-Term Reanalysis of Precipitation and the Contribution of Bias Correction to the Reduction of Uncertainty over South Korea: A Composite Gamma-Pareto Distribution Approach to the Bias Correction" by Dong-lk Kim et al.
}

\section{Anonymous Referee \#3}

Received and published: 18 May 2018

The goal of this study is to present and evaluate a bias correction of the ECMWF ERA20c reanalysis for South Korea. The authors apply a combination of transfer functions and wet frequency adjustment methods to correct the bias present in the precipitation time series. Parameters of the obtained transfer functions derived from the relation between the reanalysis grid and observed rain gauge precipitation are interpolated in space to full grid precipitation data. Overall evaluation: This is a potentially interesting paper, but in order to be published a major revision is required. The results presented

Printer-friendly version

Discussion paper 
in this paper are relatively simple and lack of deep analysis. The authors provide a long text on motivation for bias correction but omit the discussion of the bias correction in context of downscaling and do not discuss the constrains and limitations of the parameter interpolation. The authors claim in the title reduction of uncertainty but do not prove that this is the case.

1. Overall I am left unclear on the core contribution of the paper. The evaluation of the ERA precipitation over South Korea is a valuable contribution but it is very short. The applied bias correction is described in detail but a justification is missing. Finally, the spatial interpolation is not correctly validated.

2. Reviewers \#1 and \#2 provide excellent recommendations and there is no need to repeat them here. Along the lines outlined there the manuscript can be improved.

The authors may consider to rename Section 2 to "Material and methods" and to describe in addition to sections 2.1 and 2.2 in two new sections 2.3. and 2.4 the BC and the downscaling issues, possibly with some text from the introduction in which the scientific goals of the study should be clearly identified. Based on the findings and constrains discussed in this section the applied methodology can be justified and presented in detail in section 3. "Applied methodology". The validation procedure should include an analysis and discussion of the differences between the calculated and observed values at each station when this station is not included into the derivation of the interpolated parameters. This will help to access the possible errors at ungauged grid cells and thus help to judge the entire applied procedure and draw correct conclusions.

3. As a minimum requirement before revision, the manuscript has to be professionally revised and edited to correct the language and to remove the unnecessary text repetitions

Printer-friendly version

Specific:

p. 9 I. 1 - What is the rationale for using stations $4,16,28,40$ ?

Discussion paper 
- Large deviations are also visible in spring

p.9 I. 3 - The bias extreme is proportional ... I cannot see this, and even would argue that the maximum rain at station 4 is a mistake in station reading

p.9 I. 6 -This paragraph is supposed to summarize the section 2.2, but after the summary it introduces a new investigated item: wet-day. This should be presented after line 5 on page 3 . Also, I suggest to add a short description of the applied evaluation statistics after the introduction of ERA-20c.

p.9 I. 9 What is the role of climate models here ?

p.9. I. 12 Explain wet-day ( $R R>1 \mathrm{~mm} / \mathrm{d}$ ?)

p. 3 I. 21 This paragraph is an unnecessary repetition of the summary in the last paragraph.

p.12 I. 12-20 Some explanation of AIC and BIC and discussion why DGP was chosen is needed here. I cannot understand the title and the content of Table 2.

p. 13 I. 5 "Again, ..." repeats line 3

p. $15 \mathrm{I}$. 5 "I ... the suitability ..." for what? This goal of the study has not been mentioned I the introduction.

p. 15 I. 19 "... leave-one-out procedure.. " The procedure definitely needs a longer explanation and discussion. Usually one period is used for training and an another for validation.

p. 16 I. 6 Where is section 3.4.1 ?. I my opinion the section "Evaluation criteria "should be in section 2. Material and methods

p. $17 \mathrm{I} .1$ As illustrated in the previous section... The range $0 .-4.66$ is not mentioned in the previous section.

Printer-friendly version

p. 17 I. 5 What is "the degree of bias" ? 
p. 17 I. 6 "... significantly varied. .." add some numbers here to quantify this variation

p.17 I. 21. "This study introduces ..." rewrite to This study applies

p. 19, I. 10-13 "In other words.. " This is trivial. If there is no difference between model and observation then there is no need for a bias correction

p. 21 I. 16 "The bias correction ... improved the quality ..." Perhaps the mean over the region. What can be said for ungauged regions?

Figure 1. Indicate the location of the gauges $4,16,28$, and 40 used in evaluation

Interactive comment on Hydrol. Earth Syst. Sci. Discuss., https://doi.org/10.5194/hess-201836, 2018. 